\title{
The Swift short gamma-ray burst rate density: prospects for detecting binary neutron star mergers by aLIGO
}

\author{
David M. Coward ${ }^{* 1,2}$, Eric Howell ${ }^{1}$, Tsvi Piran ${ }^{3}$, Giulia Stratta ${ }^{4}$, Marica Branchesi ${ }^{5,6}$, \\ Omer Bromberg $^{3}$, Bruce Gendre ${ }^{4,8}$, Ronald Burman ${ }^{1}$ and Dafne Guetta ${ }^{7,8}$ \\ ${ }^{1}$ School of Physics, University of Western Australia, Crawley WA 6009, Australia \\ ${ }^{2}$ Australian Research Council Future Fellow \\ ${ }^{3}$ Racah Institute of Physics, The Hebrew University, Jerusalem 91904, Israel \\ ${ }^{4}$ ASI Science Data Center, via Galileo Galilei, 00044 Frascati (RM), Italy \\ ${ }^{5}$ DiSBeF - Università degli Studi di Urbino 'Carlo Bo', I-61029 Urbino, Italy \\ ${ }^{6}$ INFN, Sezione di Firenze, I-50019 Sesto Fiorentino, Italy \\ ${ }^{7}$ Department of Physics and Optical Engineering, ORT Braude, P.O. Box 78, Karmiel, Israel \\ ${ }^{8}$ INAF - Osservatorio Astronomico di Roma, Via Frascati 33, I-00040 Monteporzio Catone \\ (Roma), Italy \\ E-mail: david.cowardeuwa.edu.au, eric.howell@uwa.edu.au, \\ tsviephys.huji.ac.il, strattaeasdc.asi.it, \\ marica.branchesi@uniurb.it, omer@wise.tau.ac.il, \\ bruce.gendre@asdc.asi.it, ron.burman@uwa.edu.au, \\ dafne.guettaeoa-roma.inaf.it
}

\begin{abstract}
Presently only $30 \%$ of short gamma ray bursts (SGRBs) have accurate redshifts, and this sample is highly biased by the limited sensitivity of Swift to detect SGRBs. We account for the dominant biases to calculate a realistic SGRB rate density out to $z=0.5$ using the Swift sample of peak fluxes, redshifts, and those SGRBs with a beaming angle constraint from X-ray/optical observations. Assuming a significant fraction of binary neutron star mergers produce SGRBs, we calculate lower and upper detection rate limits of (1-180) per Yr by an advanced LIGO and Virgo coincidence search. Our detection rate is compatible with extrapolations using Galactic pulsar observations and population synthesis.
\end{abstract}

Gamma-Ray Bursts 2012 Conference -GRB2012,

May 07-11, 2012

Munich, Germany

\footnotetext{
* Speaker.
} 


\section{Introduction}

Binary neutron star mergers (NS-NS) are favoured as the progenitors for short gamma ray bursts (SGRBs), based on the association of some SGRBs with an older stellar population, and host galaxy types [1,2]. Kicks imparted to NSs at birth will produce velocities of several hundred $\mathrm{km} \mathrm{s}^{-1}$, implying that binary inspiraling systems may occur far from their site of origin. Fong, Berger \& Fox [3] using Hubble Space Telescope observations to measure SGRB-galaxy offsets, find the offset distribution compares favourably with the predicted distribution for NS-NS binaries.

Within 5 years, co-ordinated gamma-ray, X-ray, optical and gravitational-wave observations may allow the strong gravity regime of the central engine of compact object mergers to be probed. Such 'multi-messenger' observations provide the opportunity to probe these events across a vast energy spectrum, and to constrain the progenitor populations of SGRBs. Furthermore, co-ordinated optical and gravitational-wave searches may play an important role in confirming the first direct gravitational-wave observations of compact object mergers [9]. It is becoming increasingly important to constrain the rate of compact object mergers and their proposed optical counterparts in the context of up-coming gravitational-wave searches.

We calculate a beaming-corrected SGRB rate density using the Swift sample of SGRB peak fluxes, redshifts and inferred beaming angles from X-ray observations. For our GRB selection criteria we use the Jochen Greiner catalogue of localized GRBs (see Table 1) and select bursts indicated as short that have reliable redshifts up to 2012 April. We avoid using a SGRB luminosity function, models for progenitor rate evolution, and a beaming angle distribution, all of which have large uncertainties. Instead, we focus on observed and measured parameters that take into account selection effects that modify Swift's detection sensitivity to SGRBs. Finally, we use our SGRB rate density estimates to infer a detection rate of binary NS mergers by advanced LIGO (aLIGO) and Virgo (AdV) interferometers. Despite the poor statistics, this approach gives meaningful results and can be followed up when a larger sample of SGRB observations becomes available.

\section{Model}

The intrinsic SGRB rate is calculated taking into account the following observational bias effects using the sample in Table 1. GRB051221A is the only SGRB with a directly measured beaming angle in the sample (only a lower limit for the beaming angle was obtained for GRB 070724A-see Coward et al. [4]). We evaluated an upper SGRB rate density by using the smallest observed beaming angle in the sample ( $7 \mathrm{deg}$ ) and a lower rate by assuming isotropic emission.

The low energy detection bandwidth of Swift (15-150 keV) in comparison with BATSE's 50$300 \mathrm{keV}$ results in a bias against SGRBs which typically have harder emissions. Secondly, the Swift detection threshold is not simply defined by the detector sensitivity, but by a complex triggering algorithm [5]. Both these effects manifest as a bias against Swift detecting SGRBs; i.e. a smaller proportion of bursts has been detected by Swift ( 10\%) [6]. The latter effect results from the detection process employed by BAT, the Swift coded-aperture mask $\gamma$-ray detector. In addition to requiring an increased photon count rate above background (the sole triggering criterion used for BATSE), BAT employs a second stage in which an image is formed by accumulating counts for up to $26 \mathrm{~s}$ [7]. 


\begin{tabular}{lcccc}
\hline \hline SGRB & $\begin{array}{c}T_{90} \\
(\mathrm{~s})\end{array}$ & $\begin{array}{c}\theta_{\mathrm{j}} \\
(\mathrm{deg})\end{array}$ & $z$ & $\begin{array}{c}\text { peak flux } \\
\left(\mathrm{ph} \mathrm{s}^{-1} \mathrm{~cm}^{-2}\right)\end{array}$ \\
\hline \hline 101219A* & 0.6 & - & 0.718 & 4.1 \\
$100117 \mathrm{~A} *$ & 0.3 & - & 0.92 & 2.9 \\
$090510 \mathrm{~A} *$ & 0.3 & - & 0.903 & 9.7 \\
$080905 \mathrm{~A}^{a}$ & 1.0 & - & 0.122 & 6.0 \\
$070724 \mathrm{~A}$ & 0.4 & $>11^{\dagger}$ & 0.457 & 2.0 \\
$061217 \mathrm{~A}$ & 0.2 & - & 0.827 & 2.4 \\
$051221 \mathrm{~A} *$ & 1.4 & 7 & 0.547 & 12.0 \\
$050509 \mathrm{~B}$ & 0.73 & - & 0.225 & 3.7 \\
\hline \hline
\end{tabular}

Table 1: SGRB peak fluxes, $T_{90}$ and redshifts taken from the Swift online catalogue and http://www.mpe.mpg.de/ jicg/grbgen.html used to calculate Poisson rates. We use the 20-ms peak photon fluxes from the BAT2 catalogue where possible - those marked by $*$ are 1-s peak photon fluxes. ${ }^{\dagger}$ We set a lower limit on the jet opening angle from the time when the Swift XRT monitoring stopped. ${ }^{a}$ The proposed host galaxy at $z=0.1218$ for GRB 080905A [10] is a strong outlier to the Yonetoku relation [11] and a redshift $z>0.8$ would make it consistent [12]. See [4] for expanded table with references.

We attempt to crudely correct for these biases by using the observed SGRB rate from BATSE as a rate calibration for the Swift SGRB rate. Because BATSE operated at different energy thresholds and trigger sensitivities [7], for consistency we take all BATSE SGRBs with 64-ms peak flux when the trigger threshold was set to $5.5 \sigma$ in the 50-300 keV energy range (total live operation time of 3.5 years). This yields 32 SGRBs sr${ }^{-1} \mathrm{yr}^{-1}$, assuming an effective BATSE FoV of $\pi \mathrm{sr}$ [8].

Because SGRBs occur over a short duration, it is more difficult (compared to long bursts) to produce a significant signal above background. Hence instead of using the theoretical BAT sensitivity of $F_{\text {Lim }}=0.4 \mathrm{ph} \mathrm{s}^{-1} \mathrm{~cm}^{-2}$ [9], we employ a flux limit of $1.5 \mathrm{ph} \mathrm{s}^{-1} \mathrm{~cm}^{-2}$, using the smallest 20-ms peak flux from the SGRB data.

Taking into account the sensitivity reduction and k-correction (see [4] for a derivation of the k-correction), the SGRB all-sky rate can be inferred from the flux-limited SGRB sample in Table 1. We calculate the maximum distance $d_{\max }$, with corresponding redshift $z_{\max }$, that a burst at luminosity distance $d_{L}$ could be detected given Swift's sensitivity $F_{\text {Lim }}$ (see [4] for the derivation of $\left.z_{\max }\right)$.

The corresponding maximum SGRB detection volume for each burst is defined as

$$
V_{\max }=\int_{0}^{z_{\max }} \frac{d V}{d z} d z
$$

where the volume element, $d V / d z$, and luminosity distance, $d_{L}(z)$, are calculated using a flat- $\Lambda$ cosmology with $H_{0}=71 \mathrm{~km} \mathrm{~s}^{-1} \mathrm{Mpc}^{-1}, \Omega_{M}=0.3$ and $\Omega_{\Lambda}=0.7$.

To calculate the intrinsic rate of SGRBs requires accounting for the beaming angle, $\theta_{\mathrm{j}}$, of the jetted burst. Equation (2.2) expresses the beaming factor used to correct for the unobserved SGRBs that are not detected because the jet is misaligned with the detector:

$$
B\left(\theta_{\mathrm{j}}\right)=\left[1-\cos \left(\theta_{\mathrm{j}}\right)\right]^{-1} .
$$




\begin{tabular}{lccc}
\hline \hline SGRB & $\mathrm{P}_{i\left(T_{90} ; \mathrm{P}_{\mathrm{L}}\right)}$ & $\begin{array}{c}\text { lower rate } \\
\mathrm{Gpc}^{-3} \mathrm{yr}^{-1}\end{array}$ & $\begin{array}{c}\text { upper rate } \\
\mathrm{Gpc}^{-3} \mathrm{yr}^{-1}\end{array}$ \\
\hline \hline 101219A & 0.79 & 0.039 & $5.3^{\dagger}$ \\
$100117 \mathrm{~A}$ & 0.89 & 0.039 & $5.2^{\dagger}$ \\
090510 & 0.89 & 0.02 & $2.7^{\dagger}$ \\
$080905 \mathrm{~A}^{a}$ & 0.72 & 4.9 & $660^{\dagger}$ \\
$070724 \mathrm{~A}$ & 0.84 & 0.77 & 140 \\
061217 & 0.89 & 0.2 & $27^{\dagger}$ \\
$051221 \mathrm{~A}$ & 0.72 & 0.026 & 3.5 \\
050509B & 0.79 & 2 & $270^{\dagger}$ \\
\hline Total rate & & $8_{-3}^{+5}$ & $1100_{-470}^{+700}$ \\
\hline \hline
\end{tabular}

Table 2: The beaming-corrected SGRB rate densities with Poisson uncertainties using the observed constraints on $\theta_{\mathrm{j}}$, and scaled by the probability $\mathrm{P}_{i\left(T_{90} ; \mathrm{P}_{\mathrm{L}}\right)}$, except GRB $100816 \mathrm{~A}$, which uses just the $T_{90}$. Lower rate estimates assume isotropic emission, and upper rates use the observed beaming angle constraints shown in Table 1 , or the smallest observed beaming angle in the sample ${ }^{\dagger}, \theta_{\mathrm{j}} \approx 7^{\circ} .{ }^{a}$ Because of the importance of GRB 080905 for the rate density, and its uncertainty (see Table 1. for caveats), we also calculate total rates excluding this burst i.e. $\left(3_{-1}^{+2}-500_{-220}^{+340}\right) \mathrm{Gpc}^{-3} \mathrm{yr}^{-1}$.

To account for the fact that only a fraction of observed SGRB have measured redshifts, we scale the rate density by the ratio of $S$ wift bursts with redshift to those without redshifts, $F_{r} \approx 8 / 39$. The time span encompassing all observations, $T \sim 6 \mathrm{yrs}$, is defined by the start of Swift observations to the time of the most recent SGRB in the sample and we account for the fractional sky coverage of Swift, $\Omega \approx 0.17$. To account for Swift's reduced sensitivity for detecting SGRBs relative to BATSE, we use the ratio of the BATSE to Swift SGRB detection rate, which we approximate as $R_{B / S}=6.7$. This converts the Swift SGRB rate to an intrinsic SGRB rate. We point out that this correction applies only to SGRB (see [4] for an analysis including SGRBs with extended emission). Finally, we use the probabilities, $\mathrm{P}_{i\left(T_{90} ; \mathrm{P}_{\mathrm{L}}\right)}$, that each burst is a non-collapsar [13, 14], and scale the rate density by these probabilities. We note that these probabilities do not affect the rate densities significantly.

Combining all detection parameters yields the Poisson SGRB rate density of a single ( $i$ th) burst, and the total rate density for $n$ bursts:

$$
R_{\mathrm{SGRB}}=\sum_{i}^{n} \frac{1}{V_{\mathrm{i}(\max )}} \frac{1}{F_{r}} \frac{1}{T} \frac{1}{\Omega} R_{B / S} B_{i}\left(\theta_{\mathrm{j}}\right) \mathrm{P}_{i\left(T_{90} ; \mathrm{P}_{\mathrm{L}}\right)} .
$$

\section{Results and summary}

We use the above SGRB rate density to infer a detection rate of binary NS mergers by advanced gravitational-wave interferometers. For aLIGO and AdV interferometer sensitivities, the horizon distance, $D_{h}$ (all sky locations and orientation averaged over) for optimal detection of a NS-NS merger in a coincidence search is about $340 \mathrm{Mpc}$ (cosmological redshift not included). For a direct comparison with [15], the detection rate is computed for a single interferometer, with $D_{h}=197$ $\mathrm{Mpc}$, and for optimal detection in a three detector coincidence search (see figure 1). Given the 


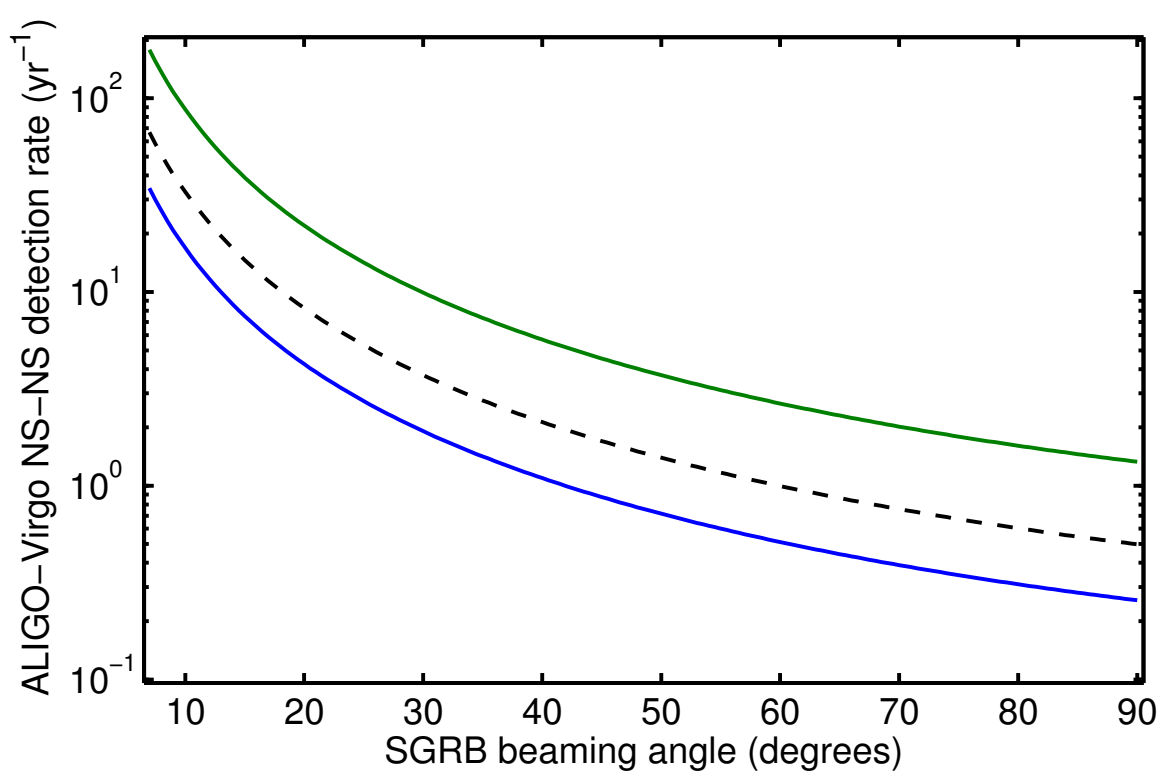

Figure 1: The lower curve plots equation 3.1, the detection rate of binary NS mergers by a single aLIGO interferometer $\left(D_{h}=197 \mathrm{Mpc}\right)$ as a function of SGRB beaming angle, using $R_{\mathrm{Low}}=8 \mathrm{Gpc}^{-3} \mathrm{yr}^{-1}$ ( $(\mathrm{see}$ Table 2). The upper curve assumes a three detector coincidence search with $D_{h}=340 \mathrm{Mpc}$. Both horizon distances used to calculate detection rates are angle averaged over all binary orientations. The dashed curve is the same as the upper curve but using the upper rate excluding GRB 080905 (see Table 1. for caveats).

uncertainty in the beaming angle distribution, we define a detection rate as a function of $\theta_{\mathrm{j}}$ using the SGRB lower rate estimate, i.e. from $\theta_{\mathrm{j}}=90^{\circ}$, scaled by $B\left(\theta_{\mathrm{j}}\right)$ and the Euclidean volume:

$$
R\left(\theta_{\mathrm{j}}\right)=\frac{4 \pi}{3} D_{h}^{3} R_{\mathrm{Low}} B\left(\theta_{\mathrm{j}}\right) .
$$

For a realistic SGRB beaming angle range $\left(\theta_{\mathrm{j}}=7-30\right)$ degrees, the corresponding detection rate by a coincidence search can range from $(10-180)$ detections per year.

The binary NS gravitational-wave detection rate estimates are based on calculating an intrinsic SGRB rate density using Swift localized bursts, taking into account dominant selection effects. This approach, based on observational data is very different from that based on Galactic binary pulsar observations and modelled population synthesis. In the latter, [15] use the observed Galactic binary pulsar population and extrapolate a NS merger rate density out to the aLIGO and AdV detection horizon. Conversely, our approach avoids this extrapolation because it is essentially an observed rate extending well beyond the average sensitivity distances of the upcoming gravitational-wave searches for compact binaries (about $300 \mathrm{Mpc}$ or $z=0.07$ for aLIGO and AdV interferometers).

In conclusion, the upcoming gravitational-wave detection era will be fundamental for resolving the SGRB-binary NS merger connection, since an unequivocal association between SGRBs and binary NS mergers will only be possible via coincident gravitational-wave and electromagnetic observations. Ultimately, a comparison beteween the SGRB rate density and the gravitational-wave detection rate will help constrain the fraction of binary NS mergers that give rise to SGRBs and the SGRB beaming angle distribution. 


\section{Acknowledgments}

D. Coward thanks the organisers of GRB12 Munich for the opportunity to present this work, and for important feedback from the conference participants and referee.

\section{References}

[1] W.H. Lee, E. Ramirez-Ruiz, J. Granot. 2005, A Compact Binary Merger Model for the Short, Hard GRB 050509b, Astrophys. J. 630, (2005), L165.

[2] Z. Zheng and E. Ramirez-Ruiz. Deducing the Lifetime of Short Gamma-Ray Burst Progenitors from Host Galaxy Demography, Astrophys. J. 665, (2007), 1220.

[3] W. Fong, E. Berger, and D.B. Fox. Hubble Space Telescope Observations of Short Gamma-Ray Burst Host Galaxies: Morphologies, Offsets, and Local Environments, Astrophys. J. 708, (2010), 9.

[4] D.M. Coward et al. The Swift short gamma-ray burst rate density: implications for binary neutron star merger rates, MNRAS submitted (arXiv:1202.2179).

[5] N. Gehrels et al. Correlations of Prompt and Afterglow Emission in Swift Long and Short Gamma-Ray Bursts, Astrophysical Journal 689 (2008) 1161.

[6] N. Gehrels, E. Ramirez-Ruiz and D. Fox Gamma-Ray Bursts in the Swift Era, Annual Review of Astronomy and Astrophysics 47 (2009) 567.

[7] D.L. Band. Postlaunch Analysis of Swift's Gamma-Ray Burst Detection Sensitivity, Astrophys. J. 644, (2006), 378.

[8] D.L. Band. Comparison of the Gamma-Ray Burst Sensitivity of Different Detectors, Astrophys. J. 588, (2003), 945.

[9] D.M. Coward et al. Towards an optimal search strategy of optical and gravitational wave emissions from binary neutron star coalescence, Monthly Notices of the Royal Astronomical Society $\mathbf{4 1 5}$, (2011), L26.

[10] A. Rowlinson, K. Wiersema, et al. Discovery of the afterglow and host galaxy of the low-redshift short GRB 080905A, Monthly Notices of the Royal Astronomical Society 408, (2010), 383.

[11] D. Yonetoku, T. Murakami, T. Nakamura, R. Yamazaki, A.K. Inoue, and K. Ioka. Gamma-Ray Burst Formation Rate Inferred from the Spectral Peak Energy-Peak Luminosity Relation, Astrophys. J. 609, (2004), 935.

[12] D. Gruber, et al. Rest-frame properties of GRBs observed by Fermi/GBM, in Proceedings of Science, Gamma-Ray Bursts 2012 Conference - GRB2012, May 07-11, 2012, Munich Germany.

[13] O. Bromberg, E. Nakar, T. Piran and R. Sari. An Observational Imprint of the Collapsar Model of Long Gamma-Ray Bursts, ApJ 749, (2012), 110 (arXiv:1111.2990).

[14] O. Bromberg E. Nakar, T. Piran R. Sari. New insights on the duration distribution of long GRBs from Collapsars (arXiv:1112.5949).

[15] J. Abadie et al. (LIGO Scientific Collaboration). Predictions for the rates of compact binary coalescences observable by ground-based gravitational-wave detectors, Class. Quant. Grav. 27, (2010), 173001. 